\title{
Research on Hexapod Biomimetic Robot
}

\author{
Yongbao Yuan ${ }^{1,}$, , Ligang Chen ${ }^{2, b}$ \\ ${ }^{1}$ School of Mechanical and Electrical Engineering, Qingdao binhai University, Qingdao 266555, \\ China; \\ ${ }^{2}$ School of information Engineering, Qingdao binhai University, Qingdao 266555, China. \\ A1092342220@qq.com
}

Keywords: hexapod biomimetic, robot, Embedded system.

\begin{abstract}
When the disaster approaches,such as earthquake, landslide, nuclear radiation zone, harsh environment and so on, rescuers can not access to it easily, to solve this,we designed the robot, the robot has six legs, each leg has three free degrees[1]. We adopted the embedded system and servo controller as the core controllers,embedded system controller communicates with the servo controller.It realized the human-computer interaction freely by the core controllers combined with voice recognition module, it entered the accident scene freely because of flexible and small size, it communicated with mobile terminal or computer easily because of the unique HD network camera, it searched environment and transmitted the data to the terminal, it searched the target quickly. So it completes the search and rescue mission accurately and effectively, to save time for rescue, so this robot has a very important significance and practical value in the field of various fields[2].
\end{abstract}

\section{Introduction}

The inspiration of this robot comes from the nature of the spider, and this robot imitates physical structure and mode of action of spider, it is a new type of intelligent biomimetic robot[3]. Compared with conventional wheeled robot, the whole system of this robot is complexity, and it is not only very easily to control, but also the benefit of this robot,more complex structures, a large number of servos,more degrees of freedom, to make the robot more flexible and movements more sensitive, it has the ability to move in the complex ground.This robot can always find the best foothold. That why it can very easily pass through a variety of terrain.Motion System achieves active damping even through the ground is complicated, this robot can also move steadily. With so many benefits, the hexapod biomimetic scouting robot becomes the focus of the study in the many fields of robotics.

\section{The overall design of robot}

The overall block diagram. The hexapod biomimetic robot using bionics design,the whole structure imitating the spider from the nature. Structure is compact and action is very sensitive[4]. 18 degrees of freedom, embedded system controller communicates with the servo controller, it can achieve human-computer interaction through the voice module, the robot system is equipped with high-definition cameras and obstacle avoidance photoelectric sensor. Multiple degrees of freedom of design to ensure that the robot can do more action, when the road condition is complex it still can complete the reconnaissance mission. Considering the robot will enter bad working environment, the body choose a very strong material,made of shell, frame and legs are high-strength alloy material, so it has a big load, the whole system is sturdy and durable. The overall block diagram of system as show in Fig 1. 


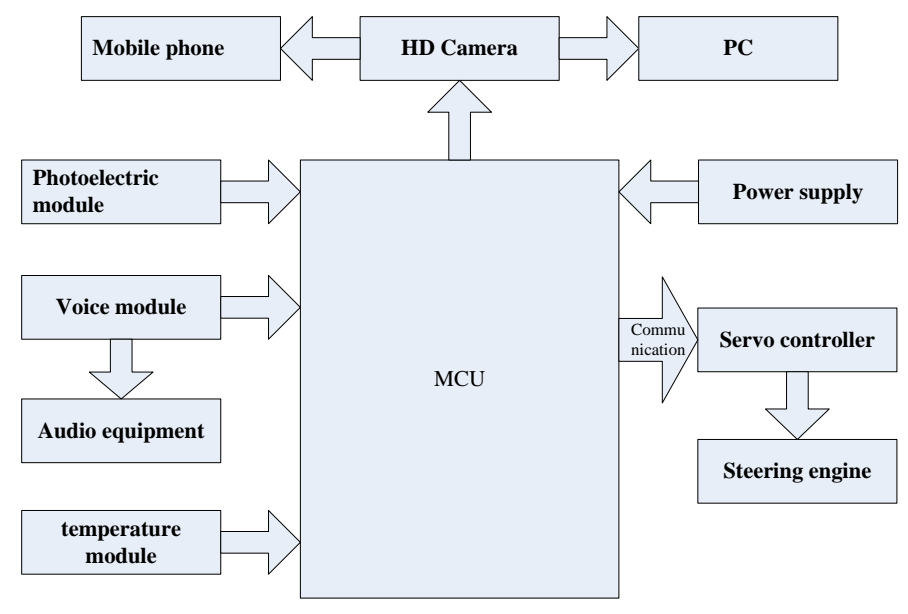

Fig 1. The overall block diagram of system

The tripod gait. In order to make the robot action smoothly, The robot uses triangle gait of spider action mode, the six legs are divided into two groups, Two groups can alternate action,then, The realization of spiders of different movements[5]. The chart of tripod gait as show in Fig 2.

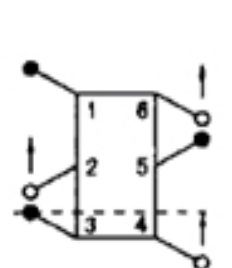

(a)

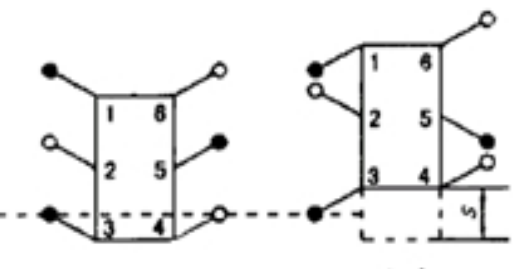

(b) (c)

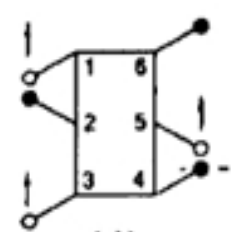

(d)

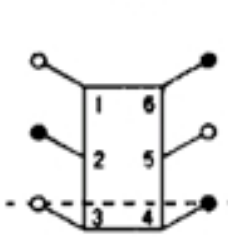

(e)

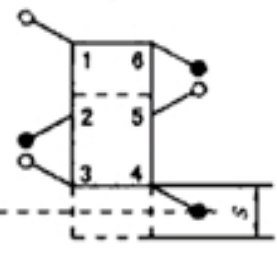

(f)

Fig 2. The decomposition chart of tripod gait

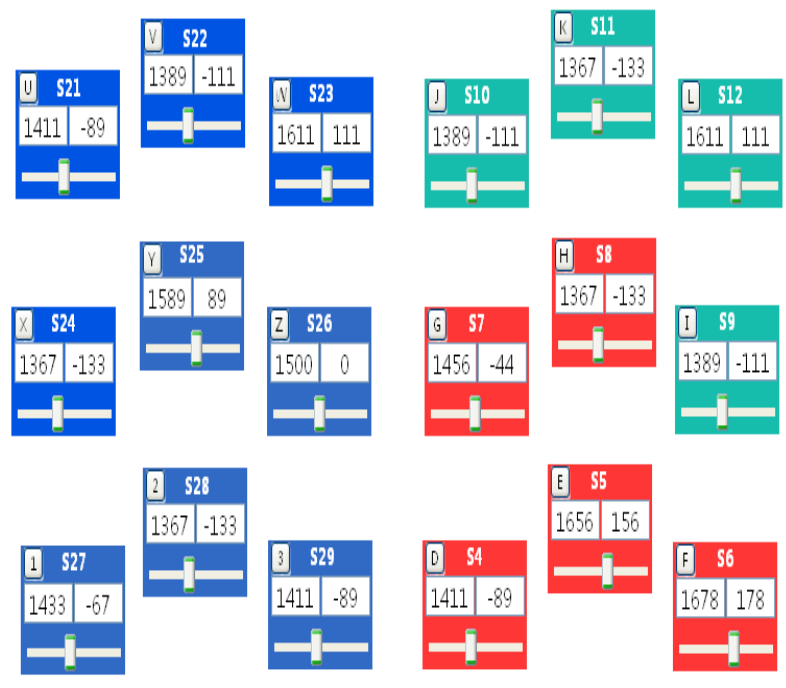

Fig 3. The chart of servo position

The main function of robot. In recent years, according to natural or man-made disasters, especially in the earthquake, disaster,radiation, nuclear leakage accident, according to this reality, we 
designed and produced the hexapod biomimetic robot[6]. Its main function is to assist the rescue workers search for trapped victims disaster site. The robot can search in the narrow space of the earthquake and mine disaster scene.

\section{The design of hardware}

Using the embedded system as the core to control the robot.The robot adopts the embedded system and servo controller communicate with each other. The robot is a stable supply of electricity, servo controller provides stable signal, the interference signal is small the robot is stable, you can set the 400 action groups. The chart of servo position as show in Fig 3. Using the ASR M08-A speech recognition module, it is supplied by $5-8 \mathrm{v}$ power. The communication between the voice module and embedded system,line out Port of the voice module is taken as the output power amplifier interface.

The leg structure of robot. A leg of the robot structure of CAD diagram as follows Fig 4:

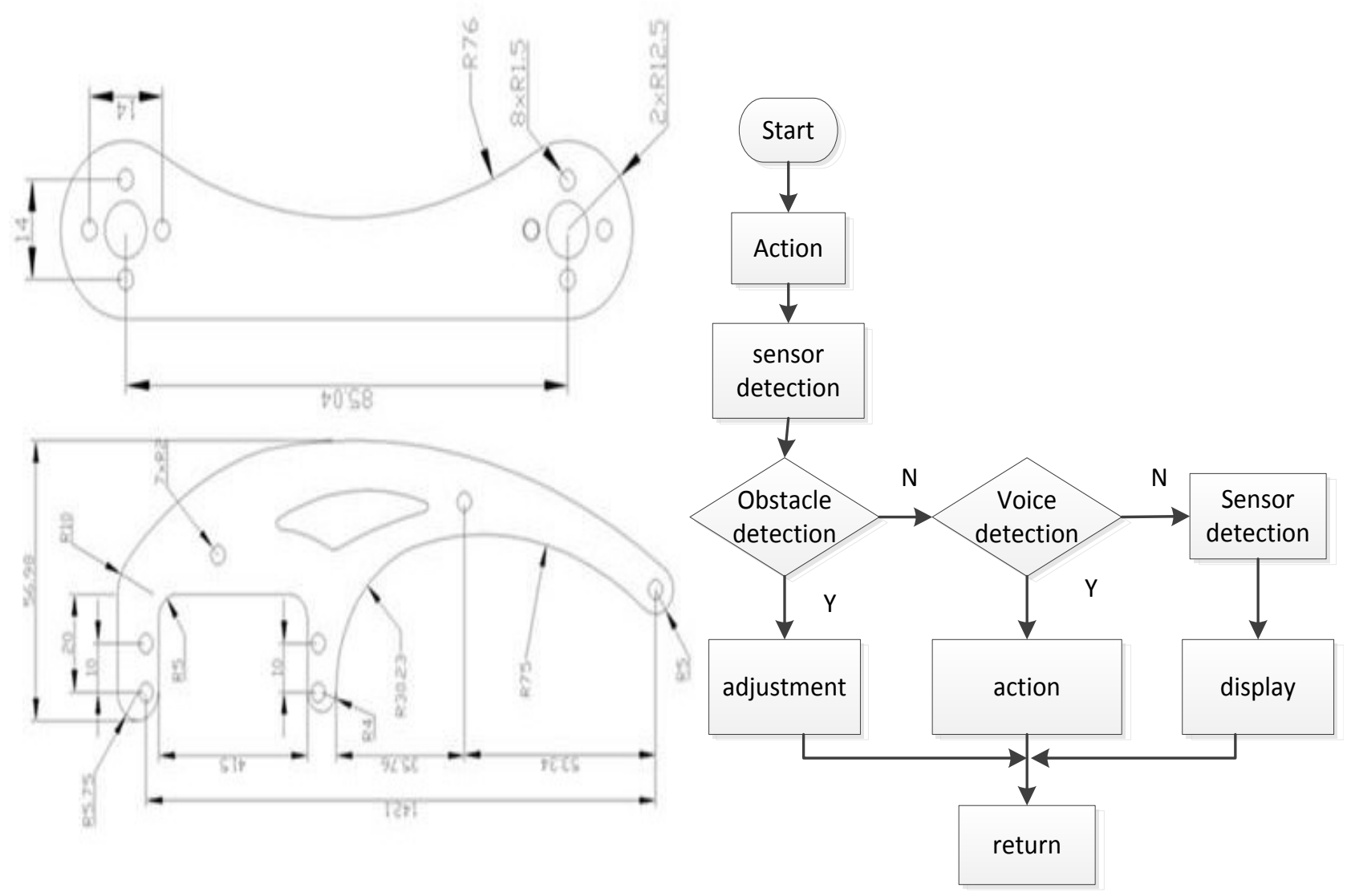

Fig 4.The CAD of robot led

Fig 5. The flow chart of software

\section{The design of software}

The hexapod biomimetic scouting robot uses building block design. The flow chart of the system is shown in the following Fig 5.

\section{Function test}

The hexapod biomimetic robot relates to many fields, the quality of hardware is reliable, and its performance is stable. The skeleton of the robot is made of high strength alloy, this could make itself more lightly. At the same time, it could improve the capacity of load-bearing and the ability of keep smooth. After the test, the robot can not only move on the flat road, but also to adapt to the rugged environment, such as, we put it on the road of after the earthquake which full of crushed stones. It 
could work very well. The work of hardware is good and it could reduce vibration, it can maintain the normal work in the case of relatively rough, and it did not happen some abnormal conditions. The robot is equipped with two photoelectric sensors, it could avoid obstacle easily. It is equipped with HD network camera in front of the robot. It is connected with mobile phone by WiFi, the phone can control the camera to realize rotating freely in the range of level of 270 degrees and vertical of 90 degrees, it is equipped with the voice module. After debugging, the robot can achieve the desired function and achieve the expected goal.The table of test as follows:

Table 1 The test of hexapod robot

\begin{tabular}{cccccc}
\hline & First & second & third & fourth & fifth \\
\hline $\begin{array}{c}\text { Obstacle } \\
\text { detection }\end{array}$ & sensitive & sensitive & sensitive & sensitive & sensitive \\
Voice & Normal & Normal & Normal & Normal & Normal \\
detection & reaction & reaction & reaction & reaction & reaction \\
Sensor & Normal & Normal & Normal & Normal & Normal \\
detection & display & display & display & display & display \\
\hline
\end{tabular}

\section{Summary}

The hexapod biomimetic scouting robot are widely application prospect in the harsh environment, major accident scene, Relief workers can not access to it easily. To improve the efficiency of Emergency rescue and disaster relief.

We designed and implemented the action of robot gait according to the triangle gait of hexapod robot. The robot's movements are flexible stable and reliable.To Solve the communication of embedded system and servo controller.We elaborate the flexibility to control the action of robot through a variety of sensor module. We reduced the weight of robot through the use of strong and lightweight alloy materials, to make the structure more compact. To increase the time of robot in operation when the battery is fully charged, to win precious time for the rescue mission.

\section{References}

[1] R.A.Brooks.A Robot that Walks:Emergent Behaviors from a Carefully Evolved Network.Neural Computation.1989,253-262.

[2] T.G.Barnes,T.Q.Truong,G.Adams.Large Deflection Analysis of a Biomimetic Lobster Robot Antenna Due to Contact and Flow.Journal of Applied Mechanics.2001,68(6):948-951.

[3] B.Klaassen,R.Linnemann,D.Spenneberg.Biologically Inspired Robot Design and Modeling.International Conference on Advanced Robotics,Coimbra,Portugal,2003,576-581.

[4] B.Klaassen,R.Linnemann,D.Spenneberg.Biomimetic Walking Robot SCORPION:Control and Modeling.Robotics and Autonomous Systems.2002,41:69-76.

[5] J.Albiezl,K.Berns.Biological Inspired Walking--How Much Nature do We Need.The International Conference on Climbing and Walking Robots,Madrid,Spain,2004,357-364.

[6] Information on http://www.robot-china.com/ 